\title{
Abundancia relativa, frecuencia y riqueza de plantas vasculares a escala local. Metodología de Índices de Ocupación de la Flora (aplicada a La Alcarria Occidental) \\ Relative abundance, frequency and richness of vascular plantas at local scale: Method for performing Floral Cover Indices (applied to Western «La Alcarria». Spain)
}

\author{
Juan Javier García-Abad Alonso ${ }^{1}$
}

\section{INTRODUCCIÓN}

La riqueza de especies vasculares depende de la extensión del territorio abarcado, pues la teoría biogeográfica plantea como hipótesis que cuanto mayor sea aquél, más aumentará la geodiversidad y, con ello, la disponibilidad de recursos para las plantas (Gaston and Blackburn, 2000). Además, concurrentemente, se amplían la heterogeneidad ambiental y los casos y grados de transformación humana del paisaje, lo que coadyuva también a alterar y enriquecer aquella diversidad biológica (Nichols et al., 1998; McKinney, 2002; Araújo, 2003; Gabriel et al., 2005; Blondel et al., 2010). Cuando a esta cuestión se unen conceptos teóricos y metodológicos tradicionales en Biogeografía y Ecología, pero vigentes por su actualidad de uso y aplicación (abundancia, áreas de distribución, diversidad/riqueza, escalas, forma y tamaño de unidades de base, frecuencia, gradientes, límites, rareza, entre otros); nos encontramos ante una problemática aún candente pese a las múltiples propuestas y soluciones metodológicas adoptadas a diferentes escalas (Preston, 1948; Brown et al., 1996; Dale, 1999; Hui and Mc-

\footnotetext{
1 Departamento de Geología, Geografía y Medio Ambiente. Universidad de Alcalá. juanj.garciaabad@uah.es
} 
Geoch, 2007; Qian and Ricklefs, 2007; Maestre et al., 2008; Wilson, 2008; Gaston and Fuller, 2009; Jiménez-Alfaro, 2009; Grillo and Gianfranco, 2011; entre muchísimas otras más). Este trabajo trata sobre tales cuestiones a escala local.

Un método de inventario de la flora que utiliza como unidad mínima de información la cuadrícula UTM $1 \times 1 \mathrm{~km}$ ha sido aplicado en dos cuadrículas UTM de $10 \times 10 \mathrm{~km}$ (ED50). Ambas pertenecen a una misma subregión natural (La Alcarria Occidental), pero están situadas en dos sectores diferentes de la misma: 30TWL02 (Utande), en la provincia de Guadalajara, en la Alta Alcarria; y 30TVK86 (Ambite), en las provincias de Madrid y Guadalajara, en la Baja Alcarria (figura 1).

FIGURA 1

MAPAS DE SITUACIÓN DE LAS DOS CUADRÍCULAS ESTUDIADAS DENTRO DE LA ALCARRIA OCCIDENTAL, Y DE ÉSTA DENTRO DE LA ESPAÑA PENINSULAR

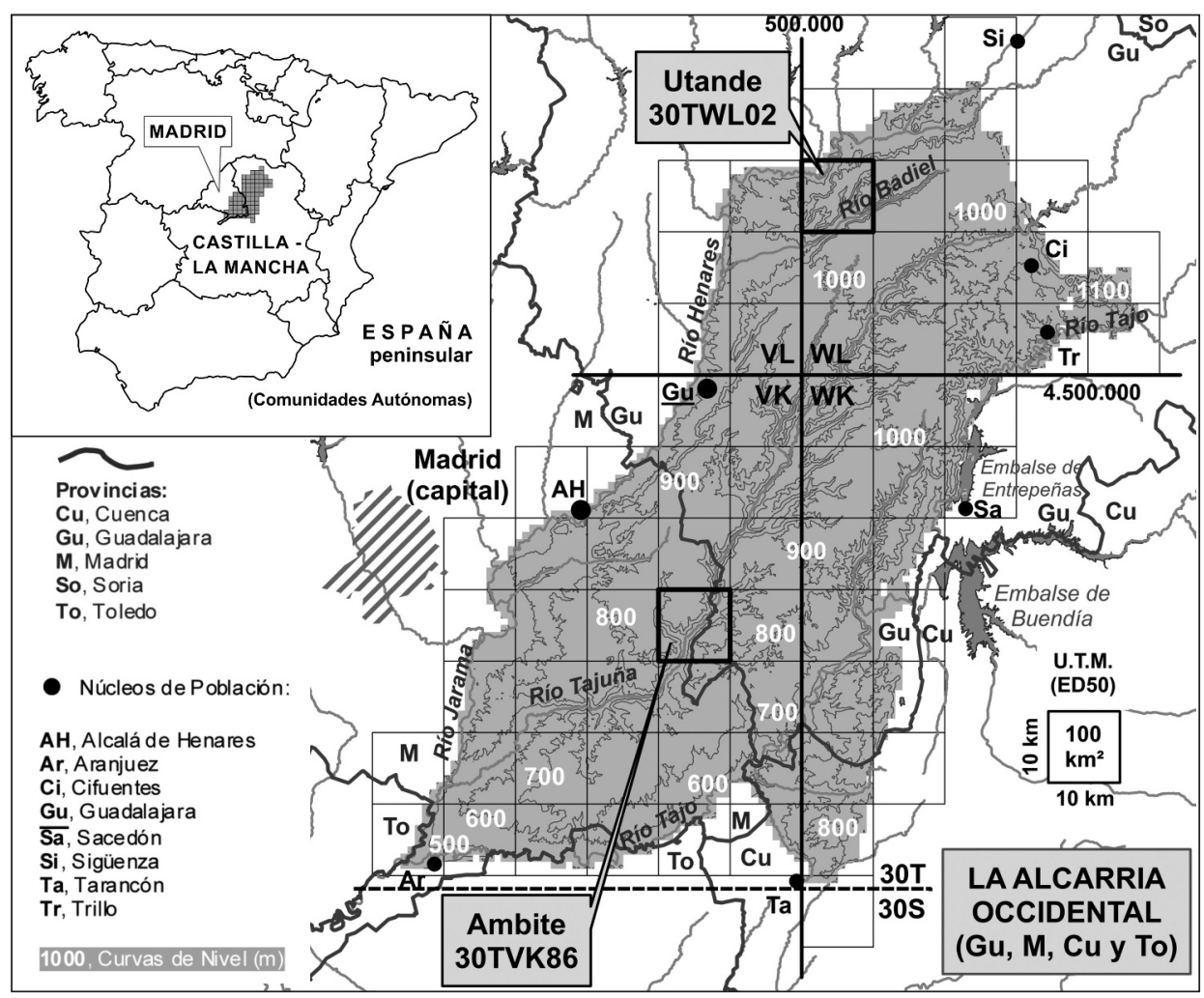

Fuente: Elaboración propia.

Estudios Geográficos, Vol. LXXVI, 279, pp. 499-530, julio-diciembre 2015

ISSN: 0014-1496, eISSN: 1988-8546, doi: 10.3989/estgeogr.201518 


\section{CUADRo 1}

SIGNIFICADO DE LOS ACRÓNIMOS Y ABREVIATURAS QUE APARECEN EN EL TEXTO

1C: Cuadrícula UTM de $1 \mathrm{~km}$ de lado, $1 \times 1 \mathrm{~km}$ ó 1 km²

4C: Cuadrícula UTM de $2 \mathrm{~km}$ de lado, $2 \times 2 \mathrm{~km}$ ó 4 km²

25C: Cuadrícula UTM de $5 \mathrm{~km}$ de lado, $5 \times 5 \mathrm{~km}$ ó $25 \mathrm{~km}^{2}$

100C: Cuadrícula UTM de $10 \mathrm{~km}$ de lado, $10 \times 10 \mathrm{~km}$ ó 100 km²

AR: Abundancia relativa

ED50: Datum Europeo de 1950, sistema geodésico de referencia covigente en España hasta el 31 de Diciembre de 2014

ETC: Entidad taxonómica cartografiable (ya sea taxón o agrupación de taxones)

GPS: Sistema Global Positioning System de localización mediante satélites

IOF: Índice de Ocupación de la Flora

IOP: Índice de Ocupación de la Planta

No PVR: No permanentemente visible y reconocible (a lo largo del año)

Periodo A: Abril y mayo

Periodo B: Junio y julio

Periodo C: Agosto y septiembre

Periodo D: Octubre, noviembre, diciembre, enero, febrero y marzo

PVR: Permanentemente visible y reconocible (referido a una planta o taxón)

subsp.: subespecie

spp.: varias especies del mismo género

UTM: Sistema de coordenadas planas Universal Transverse Mercator

Los propósitos de tal labor han sido conocer para ambos territorios: a) la riqueza florística más amplia posible; b) la distribución geográfica detallada de las plantas, expresada en forma de Cartografía corológica; c) la abundancia relativa y frecuencia de cada una de ellas; y d) su autoecología general. Con ello se puede hacer una comparación fitogeográfica altamente informada de ambos espacios. El contexto y antecedentes del método, los postulados generales y algunos resultados previos se encuentran en Nuet y Panareda (1991-93), Panareda (1996), Meaza (2000), García-Abad (2004, 2006, 2009) y GarcíaAbad et al. (2007, 2009), entre otras publicaciones.

Así pues, en este trabajo se adopta un sistema de rastreo florístico sistemático que integra tres presupuestos: a) un formato territorial de $100 \mathrm{~km}^{2}$ convencional, regular y normalizado en cuanto a forma y tamaño; b) una resolución espacial detallada de $1 \mathrm{~km}^{2}$, lo que implica prospectar 100 subunidades 
regulares estandarizadas; y c) añadir la abundancia relativa de la planta a los datos de simple presencia en cada recinto UTM de $1 \times 1 \mathrm{~km}$. Casi similar por el cumplimiento de las dos últimas condiciones fue el trabajo de Nuet y Panareda (1991-93) sobre Montserrat, que supone el antecedente directo del aquí presentado y en el que nos hemos inspirado.

Los objetivos específicos de este artículo son: a) presentar el método de inventario florístico, modificando levemente y ampliando aspectos y comentarios a los expuestos en García-Abad (2009) y García-Abad et al. (2007 y 2009); b) formular y aplicar dos Índices de ocupación florística (IOP e IOF); c) presentar una síntesis cuantitativa de la riqueza y semejanza florísticas de ambas cuadrículas; y d) exponer el Catálogo de plantas de uno de los dos contingentes florísticos diferenciados, con sus particulares referencias cuantitativas; destacando algunas plantas singulares.

En un segundo artículo que aparecerá en el siguiente número de esta Revista, se expondrá una ampliación con más resultados y casos concretos de mapas de distribución.

METOdOlOGía DE INVENTARIO FLORÍSTICO CON FINES GEOGRÁFICOS A ESCALA LOCAL

La información de partida se recaba a partir de un método de inventario sencillo (García-Abad, 2009; García-Abad et al., 2007 y 2009), pero que implica bastantes cuestiones que deben cuidarse con rigor y cuya exposición se amplía y reordena en este artículo. En relación con los diferentes aspectos a considerar (divididos en epígrafes), el inventario consiste en las asunciones, condiciones, criterios y requisitos siguientes (se numeran correlativamente, para facilitar su identificación y referencia):

\section{Tamaño y forma de las unidades a escala local y resolución espacial detallada}

1) El área de estudio a inventariar tiene la forma de un cuadrado según el sistema cartográfico UTM (ED50).

2) El tamaño de dicha área es de $100 \mathrm{~km}^{2}$ (Cuadrícula de $10 \mathrm{~km}$ de lado). Para abreviar, llamaremos $100 \mathrm{C}$ a partir de ahora a esta unidad territorial.

3) El área se divide en cien subunidades de $1 \mathrm{~km}^{2}$ ( $1 \mathrm{~km}$ de lado). Éstas constituyen la unidad mínima de inventario y, por tanto, de informa- 
ción florística. A su vez, por fusión regular de éstas, el área de estudio se puede dividir en otros dos agregados mayores que la abarcan ajustada y enteramente: veinticinco subunidades de $4 \mathrm{~km}^{2}(2 \mathrm{~km}$ de lado) y cuatro subunidades de $25 \mathrm{~km}^{2}$ (5 km de lado). A partir de ahora llamaremos a estas subunidades 1C, 4C y 25C, respectivamente.

\section{Presencia de plantas, reconocimiento y anotaciones relacionadas}

4) Se inventarían por avistamiento y reconocimiento directo las plantas vasculares presentes en cada unidad de información básica:

4a) Todas las plantas espontáneas (autóctonas y alóctonas, ya sean naturalizadas o adventicias) salvo los pleustófitos, pues estas plantas acuáticas requieren una especial estrategia de inventario.

4b) Plantas cultivadas solo cuando son leñosas o vivaces grandes, debido a su carácter permanente y fácilmente visible en el paisaje vegetal. Por contra, no se inventarían las herbáceas anuales directamente cultivadas por su permanencia cíclica y/o coyuntural y no necesariamente continuada Sin embargo, dada la espontaneidad invocada en 4a), sí se inventarían estas plantas cuando aparecen escapadas o silvestres en hábitat diferentes a los campos cultivados, o en años posteriores al cese de su cultivo.

4c) Se discrimina, con anotaciones diferenciadas, su carácter espontáneo o no. A su vez, atendiendo a hechos objetivos, datos conocidos y cuando es pertinente, en el apunte de ambas alternativas se diferencia entre autóctonas y naturalizadas/adventicias, en unos casos; y entre cultivadas y asilvestradas, en otros. Puede darse el caso de dos o más anotaciones diferentes para un mismo taxón, cuando se observan caracteres diferentes.

5) Casi todas las identificaciones se hacen directamente en el lugar de prospección, con ayuda de floras manuales (Bolòs et al., 2005 y Castroviejo, 2001). De este modo, se favorece el mayor avance espacial posible en el rastreo. En casos de duda y de plantas no incluidas en esas obras, se toman muestras para determinarlas en laboratorio, empleando otras floras o catálogos con claves dicotómicas (Bolòs y Vigo, 1984-2001; Valdés et al., 1987; Castroviejo, 1986-2012; Aizpuru et al., 2000; López, 2007). 
6) Se toman datos del estado fenológico medio de cada taxón, así como otros ecológicos y geográficos básicos de donde se encuentran individuos o poblaciones.

7) Se anotan localizaciones precisas, mediante GPS, de ejemplares o poblaciones singulares, por su rareza, interés biogeográfico, mesológico, de conservación, etc.

8) Además de tomar muestras de plantas para despejar dudas taxonómicas, se recogen muestras vegetales permitidas para intentar dar testimonio del mayor número posible de taxones. Su depósito se está efectuando en el AH Herbarium (Universidad de Alcalá), donde pueden consultarse. Hasta el momento se han depositado más de 200 pliegos.

\section{Ámbitos a inventariar}

9) Dentro de cada $1 C$, se visitan todas las unidades de paisaje, así como todos los biótopos o hábitat posibles. Cuando son extensos, además se cubren varios tramos/sectores de los mismos. Y nunca se deja de visitar ninguno de los cuadrantes de la cuadrícula (subdivisiones de $0,5 \mathrm{~km}$ de lado, ó $0,25 \mathrm{~km}^{2}$ ). Todo ello con objeto de alcanzar el máximo avistamiento posible de fitodiversidad, pero también para estimar adecuadamente la abundancia relativa de cada taxón dentro de cada 1C.

10) No se prospectan los cascos urbanos, áreas residenciales ni, obviamente, parcelas, áreas o fincas privadas valladas (salvo cuando se pudo conseguir permiso), o cualquier recinto o lugar que tenga restricciones de acceso o implique dificultades severas.

\section{Temporalidad y fenología}

11) Cada unidad $1 C$ es visitada una sola vez, con objeto de que la prospección florística avance lo más posible en cubrimiento territorial.

11a) Se establece que una $1 C$ se visite durante todo o buena parte del periodo de luz solar de un solo día. Puede ser inferior a un día, si por la sencillez de la cuadrícula se puede completar en menos tiempo. Del mismo modo, en algunas ocasiones, si por la complejidad de la cuadrícula no basta un día, entonces se vuelve otro día inmediato para 
completarla, tratando siempre de mantener un equilibrio en el esfuerzo de prospección respecto al resto de cuadrículas.

11b) Hay que advertir, en cualquier caso, que la configuración geográfica de La Alcarria es tal que se adapta bien a esta unidad temporal para completar la prospección. Si la región fuese de otro tipo (montañosa, costera, de clima extremo, etc.), evidentemente debiera adaptarse dicha unidad temporal de inventario.

12) Las visitas se hacen en cualquier época del año:

12a) Se pretende así: a) aprovechar todos los meses del año para avanzar más rápidamente en el cubrimiento territorial, sin estar sujeto a la demora que supondría esperar las estaciones más propicias en implantación floral de un año para otro; b) cubrir todo el ciclo anual pues, aunque pocas, hay plantas de estacionalidad otoñal e, incluso, invernal (geófitos como Crocus o Gagea, etc.; plantas primo-vernales, como Veronica spp., Erophila verna, entre otros casos) que es necesario observar para conseguir listados florísticos lo más completos posible; y c) minimizar las inevitables demoras que supondrían las contingencias meteorológicas, de agenda y trabajos paralelos del prospector/-res (especialmente, cuando solo haya un único prospector).

12b) Consecuencia de esta condición es que el número de horas de luz solar no es el mismo en los diferentes meses del año. En consecuencia, la unidad temporal «día» no es uniforme en duración del número de horas. Sin embargo, la experiencia nos ha mostrado que esto no es objeción para la relación tiempo/esfuerzo/riqueza, puesto que coincide que, cuando menos horas de sol tenemos, hay menos plantas que inventariar por razones fenológicas. Por ello, da tiempo a avistar razonablemente las plantas detectables en ese periodo del año.

13) Por razones fenológicas, las visitas a las cien $1 C$ se efectúan de un modo regular distribuido tanto temporal como espacialmente.

13a) Tal regularidad consiste en agrupar cartográficamente cuatro $1 C$ en una 4C. De este modo, las cien $1 C$ del área se agrupan en veinticinco 4C (punto 3).

13b) Como en cada recinto $4 C$ hay cuatro $1 C$, dentro del año se van a considerar también cuatro periodos diferenciados de inventario, que tendrán relación con la secuencia fenológica anual y con el nivel de avistamientos posibles: periodos A (abril y mayo), B (junio y julio), C (agosto y septiembre) y D (resto). Así, dentro de una 4C, cada 1C 
se inventaría en un periodo diferente de los referidos, con objeto de completar en cada 4C el ciclo fenológico y de compensar la visibilidad potencial de las plantas. Esta normalización fenológica entre las veinticinco $4 \mathrm{C}$ posibilitará en buena medida la comparación de la riqueza florística entre ellas. Cada periodo A, B, C ó D se adjudica al azar a cada $1 C$ (figura 2 ).

FIGURA 2

EXPRESIÓN GRÁFICA DE LA ESTRATEGIA DE INVENTARIO QUE PERMITE PALIAR PARCIALMENTE LAS RESTRICCIOINES FENOLÓGICAS MEDIANTE LA VISITA ALTERNANTE DE CUADRÍCULAS DE $1 \times 1$ KM DENTRO DE CUADRÍCULAS DE $2 \times 2 \mathrm{KM}$.

$2 \mathrm{~km}$

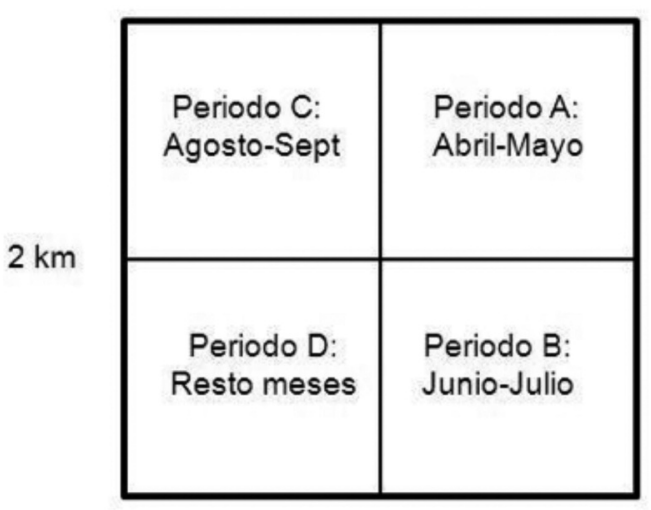

Fuente: Elaboración propia.

NOTA: Se presenta el caso de un supuesto cualquiera obtenido al azar.

13c) Se posibilita, así, el avistamiento de plantas propias de todos los periodos del año, pero empleando más tiempo/esfuerzo en los periodos de mayor implantación floral.

\section{Abundancia relativa}

14) Se apunta la Abundancia Relativa (AR, a partir de ahora) de cada planta en cada $1 C$. 
14a) El sistema está basado en Nuet y Panareda (1993) y la variante establecida después por García-Abad et al. (2009, p. 164). El aquí aplicado modifica ésta, pues elevan a cinco las categorías:

- Uno (Localizado, con muy pocos individuos). Sólo se aplica al grupo de plantas que, además de ser vivaces, mantienen durante todo el año alguna parte vegetativa que permita su identificación sin duda taxonómica seria. Son plantas fijas o permanentes en el paisaje vegetal, que se pueden ver y reconocer en cualquier época de año. Esto permite contar sin problema su número (individuos, ejemplares o matas) dentro de la cuadrícula. Se incluye en esta categoría el taxón que se encuentra en número inferior a diez. Esta información, que afecta fundamentalmente a árboles y arbustos, interesa tenerla en cuenta en las distribuciones geográficas. Cuando se avistan diez o más, pasan a la categoría «Dos» («localizado»). El resto de plantas no se cuentan y pasan directamente a esta última categoría independientemente del número de ejemplares avistados, pues esa labor sería muy ardua y muy poco relevante.

- Dos (Localizado). Oscila entre taxones simplemente presentes, desde muy raros o difíciles de encontrar, hasta otros que pueden ser de raros a habituales (incluso puntualmente frecuentes o abundantes), pero en localidades, biótopos o formaciones que ocupan espacios aproximadamente inferiores al $10 \%$ de la superficie de la cuadrícula. Con la estructura típica de los usos de suelo actuales en La Alcarria Occidental, suele ser la categoría habitual para la mayor parte de las plantas.

- Tres (Frecuente). Taxones cuya presencia es detectada con facilidad, puesto que son de habituales a abundantes en espacios que oscilan aproximadamente entre el 10 y el $30 \%$ de la superficie de la cuadrícula. Si puntualmente son muy abundantes, el límite inferior puede rebajarse hasta el $5 \%$ (carrizales, riberas, corredores, etc.). Es difícil que plantas anuales, bienales o geófitos alcancen estos niveles, porque no suelen cubrir tanto territorio; pero, no son raras las excepciones (Amaranthus spp., Avena spp., Bromus spp., Convolvulus arvensis, Papaver rhoeas, Polygonum spp., Salsola kali, etc, a veces son muy abundantes en biótopos antropizados, entre otros casos).

- Cuatro (Abundante). Taxones extensamente implantados. Son habituales y frecuentes, o abundantes, en espacios aproximadamente 
entre el 30 y el $60 \%$ de la superficie. Muy pocas plantas pueden alcanzar este nivel.

- Cinco (Muy abundante). Taxones muy extensamente implantados. Son frecuentes, o abundantes, en espacios aproximadamente superiores al $60 \%$ de la superficie. Son aún menos los casos en que puede darse esta categoría, porque el paisaje alcarreño está bastante cultivado.

14b) La AR se valora y apunta al final de la visita, una vez se ha recorrido toda la cuadrícula. Se repasan los taxones inventariados y se anota la categoría pertinente. Además de por la observación in situ, la estimación de la superficie involucrada en cada caso se hace con ayuda de la ortofotografía más reciente, que se lleva al campo como material de apoyo. Una condición fundamental para valorar esta cuestión es haber recorrido convenientemente la cuadrícula (recuérdese el punto 9).

14c) Obviamente, solo se anota la AR que se ve el día de la prospección, sin especular sobre lo que podría verse en otras épocas del año.

\section{Consideraciones metodológicas finales}

Para cumplir rigurosamente con el objetivo de establecer la distribución de las plantas, debe cuidarse la posibilidad de comparar equitativamente su presencia en los recintos territoriales (1C, 4C, 25C y 100C). Con el método propuesto, se era consciente de antemano de que ese objetivo no podía lograrse para las cien $1 C$, porque serían visitadas en diferentes periodos y, por tanto, en situaciones fenológicas no equiparables en cuanto a riqueza detectable.

Pese a estas restricciones del método, éste permite dos soluciones parciales para confeccionar cartografía equilibrada y comparable entre dos o más sectores. Una es fundir las unidades $1 C$ en las inmediatamente más grandes y de menor resolución (4C), ya expresado en 13b), para minimizar los desajustes en cuanto a la riqueza y composición florística detectadas. Y la otra solución es separar contingentes de plantas diferenciados según su implantación temporal, para reducir una parte de las restricciones aludidas. Esta última cuestión implicó primero aplicar el método $\mathrm{y}$, con los datos y experiencia recabados, elaborar después listados de plantas diferenciados. 


\section{RESULTADOS}

Los inventarios se realizaron entre 2005 y 2012. En la cuadrícula de Ambite (30TVK86) no se pudieron visitar $\operatorname{dos}^{2}$ de las cien cuadrículas de $1 \mathrm{~km}^{2}$ por estar completamente dentro de fincas privadas con acceso no permitido por vallado. En consecuencia, fueron noventa y ocho las cuadrículas de las que se pudo hacer inventario. Los resultados se exponen en apartados, según un orden progresivo de tratamiento/elaboración.

\section{Contingentes florísticos diferenciados}

Tras las observaciones, anotaciones fenológicas y comparaciones efectuadas, se dispuso de datos que permitían caracterizar las plantas por su posible detección y determinación en campo. Aunque se establecieron varios grupos en función de su biótipo, talla, durabilidad de la condición de hacerse aparente y otras consideraciones; en aras de la síntesis, se unificaron finalmente en dos grandes Grupos (García-Abad et al., 2009, p. 165). A continuación se amplían explicaciones y comentarios al respecto:

1) Grupo 1, de Plantas Permanentemente Visibles y Reconocibles (PVR, a partir de ahora) que, por ser perennes o perennizantes, cumplen dos condiciones: a) pueden ser avistadas en cualquier época del año; y b) no presentan serias dificultades para ser reconocibles siempre y determinadas taxonómicamente. En conjunto, son las plantas con más fitomasa y dan la impronta fisionómica al paisaje vegetal. Incluyen:

la) Todos los fanerófitos y caméfitos, con algunas excepciones. Éstas se deben a que algunos caméfitos no cumplen la condición de ser suficientemente visibles y, por tanto, reconocibles por su muy baja talla o raquítica visibilidad en alguna época del año. Esto puede conllevar omisiones de presencia no reales (algunos ejemplos: Anagallis monelli, Astragalus glaux, Centaurea alba, Haplophyllum linifolium, Linum narbonense, Ononis spp., Parietaria judaica, Teucrium pseudochamaepytis, Thesium humifusum,...). Otras veces, se trata de unos muy pocos fanerófitos y caméfitos que, pese a poder ser avistados siempre, no cum-

${ }^{2}$ Dominadas por bosques y monte bajo, aportarían una riqueza media-baja. Estimamos que debieron ser poquísimos los taxones que eventualmente dejaron de detectarse respecto al conjunto de la 100C. 
plen con la condición de ser siempre reconocibles taxonómicamente. Ello se debe a que presentan hibridaciones habituales, o bien una especial complejidad en la determinación relacionada con avistamientos en una época del año poco propicia para solventar claves de determinación taxonómicas- Estos casos se dan en algunas especies de Helianthemum, Limonium, Morus, Rosa, Salix, Tamarix, entre otros. Así, para evitar omisiones o errores por confusión entre taxones alternativos, hemos aplicado un criterio conservador por el cual estas plantas se incluyen en el otro contingente florístico (Grupo 2, ver más adelante).

1b) Varios hemicriptófitos vivaces o perennizantes que pueden avistarse y determinarse sin dificultad, pese a que en algún periodo del año presenten parte o gran parte de su cuerpo vegetativo deteriorado o incompleto. Normalmente tienen la propiedad de ser arrosetados, cespitosos, sufruticulosos o sufruticosos, aunque a veces son simplemente escaposos o rizomatosos.

1c) Algunos pocos geófitos que, por tener propiedades análogas a las referidas, cumplen las dos condiciones anteriores. Normalmente son cespitosos o sufruticulosos.

1d) Las lianas leñosas que cumplen las dos condiciones.

1e) Los hidrófitos o helófitos que cumplen las dos condiciones.

La propiedad fundamental de estas plantas PVR es que, al ser detectables cuando están presentes en cualquier $1 C$, su distribución y abundancia relativa pueden compararse en mapas con resolución de $1 \mathrm{~km}^{2}$, sin importar el periodo de inventario. A efectos de representar su distribución se consideran Entidades Taxonómicas Cartográficas (ETC, a partir de ahora) por sí solas.

2) Grupo 2, de Plantas No Permanentemente Visibles y Reconocibles (No PVR, a partir de ahora), formado por el resto de taxones; es decir, los terófitos y el resto de biótipos que no cumplen con aquellas dos condiciones. Además, se unen a éste las plantas exceptuadas en el Grupo 1, ya referidas.

La propiedad fundamental de estas plantas No PVR es que ni su distribución ni su abundancia pueden compararse en mapas con resolución de $1 \mathrm{~km}^{2}$. Sin embargo, sí alcanzarán cierto grado de ajuste en la comparación en mapas con resolución de $4 \mathrm{~km}^{2}$, pues cada $4 \mathrm{C}$ reúne por fusión de las cuatro $1 \mathrm{C}$ una composición florística correspondiente a todo un ciclo anual, completo fenológicamente. Es evidente que, para plantas efímeras y que sean aparentes o reconocibles algunas pocas semanas al año, ese ajuste será solo aproximado y las restricciones no del todo subsanables. Sin embargo, para la mayor parte del resto de las plantas, que pueden avistarse durante varios meses o gran parte del año, 
sus distribuciones en mapas de unidades 4C permiten una comparación sobradamente confiable. En cuanto a la comparación de su abundancia, esta estrategia aporta soluciones variables, pero parciales y en general menos confiables.

Dentro de este grupo No PVR se ha arbitrado una estrategia especial para unas pocas plantas. Su objetivo es, con fines exclusivamente cartográficos, no dejar fuera de la posible comparación en mapas de resolución de $1 \mathrm{~km}^{2}$ a algunas plantas de gran significación e implantación fija. Los casos más sencillos han consistido, simplemente, en fusionar variedades en subespecies, o variedades y subespecies en especies, para representar su distribución. Pero, en otros casos, aquélla ha consistido en efectuar algunas agrupaciones supraespecíficas (subgéneros, grex o géneros), cuando era posible y se ha creído pertinente, de taxones con una filiación filogenética muy próxima, cuyas claves de discriminación taxonómica eran dificultosas o implicaban especial detalle. De este modo, esas agrupaciones se han considerado como una sola ETC, al mismo nivel que las plantas PVR. Esta solución supone una «modificación metodológica» de la fitodiversidad a representar en esos mapas por razones meramente operativas. Se aplica a plantas que son permanentemente visibles aunque no siempre reconocibles (fanerófitos -algunos taxones de Rosa, Tamarix, etc. ${ }^{3}-$; caméfitos - «idem» Helianthemum, etc.-; hemicriptófitos - «idem» Carex, Stipa, Vicia, Viola, etc.-; entre otras plantas).

En los casos de otras plantas No PVR, esta estrategia de agrupación de taxones también se ha aplicado a plantas no siempre visibles, con objeto de conocer su distribución aproximada en mapas con resolución de $4 \mathrm{~km}^{2}$ (algunas especies de los géneros Arrenatherum, Avena, Bromus, Cuscuta, Malva, Orobanche, Polygonum, Scrophularia, Taraxacum, etc.).

Como un primer resultado del trabajo, en este artículo se expone el Catálogo de las plantas del Grupo 1 y de las agrupaciones taxonómicas referidas para ser representables con resolución de $1 \mathrm{~km}^{2}$ (Véase Anexo, al final).

\section{Diseño de Índices de Ocupación}

A partir del método de inventario, del tamaño y de la forma de las áreas de estudio, se propone la formulación de unos índices de cálculo muy sencillo.

${ }^{3}$ El caso de los sauces (Salix spp.) era complicado por lo habitual de sus hibridaciones y problemas fenológicos. Pero hemos apreciado que su cartografía con resolución de $4 \mathrm{~km}^{2}$ era suficientemente fiable y elocuente geográficamente, por lo que hemos preferido no aplicar la referida excepción operativa. A pesar de todo, reconocemos que se podría haber optado por tal alternativa. 
El primer paso consiste en asignar un valor indicativo cuantificable a las categorías de AR. Se han adoptado los siguientes: 1) Localizado con muy pocos individuos: 0,$01 ; 2$ ) Localizado: 0,$1 ; 3$ ) Frecuente: 0,25; 4) Abundante: 0,5; y 5) Muy Abundante: 1.

El segundo paso consiste en calcular el valor que posee una planta dentro de un recinto 100C, sumando todas las AR que tiene esa planta en cada 1C. Se diseña así un primer índice que se denominará Índice de Ocupación de la Planta (IOP):

$$
\mathrm{IOP}=\sum_{\mathrm{i}=1}^{100} \mathrm{AR}_{\mathrm{i}}
$$

siendo hasta 100 las unidades $1 \mathrm{C}$ inventariadas

Por tanto, referido a una planta (o a una ETC), este IOP contempla unificadamente lo siguiente: a) su presencia en un área; b) la abundancia relativa conjunta que posee en ese área; y c) la frecuencia de esa presencia en número de subunidades regulares. Ello permite, por la cuantificación de tales parámetros, ordenar las plantas/ETC de mayor a menor ocupación en ese área.

Como segundo resultado del trabajo, en el Anexo se expresan tales cuantificaciones y el orden para el grupo florístico catalogado. Algunas muestras de los datos más significativos son las siguientes: a) se ha constatado que lastón (Brachypodium retusum), aliaga (Genista scorpius) y quejigo (Quercus faginea) son las tres plantas con mayor ocupación en Utande; frente a encina (Quercus ilex), tomillo vulgar (Thymus vulgaris) y ge del campo (Helianthemum cinereum subsp. rotundifolia), en Ambite; b) se comprueba que los tres valores más altos de IOP obtenidos han sido los de lastón $(33,55)$ y aliaga $(28,67)$, en Utande; y el de encina $(24,70)$, en Ambite; y c) las plantas con la máxima frecuencia posible fueron: cardo corredor (Eryngium campestre) en ambas cuadrículas; llantén mayor (Plantago lanceolata), rosal (agrupación Rosa spp.) y zarza (Rubus ulmifolius), en Utande; y triguera (Dactylis hispanica), oreja de liebre (Phlomis lychnitis) y coscoja (Quercus coccifera), además de los ya mencionados ge del campo y encina, en Ambite.

Por el extremo opuesto de valores bajos, tanto el IOP como la frecuencia sirven como indicadores de rareza. Si, por ejemplo, se analiza la frecuencia, destaca sobremanera que de las 339 ETC mostradas en el Catálogo, 116 (Ambite) y 107 (Utande) se encuentren en el rango de estar presente entre sólo una y diez cuadrículas. Ello dota de una singularidad especial al 43,1\% (Ambite) y $36,4 \%$ (Utande) del grupo florístico catalogado. Si, además, en este 
apartado florístico se añaden otros elementos biogeográficos de singularidad propios de La Alcarria Occidental y se dejan fuera taxones referidos en otros trabajos (García-Abad, 2004 y García-Abad et al., 2009), merecen una mención especial el hallazgo de los siguientes: Asplenium trichomanes subsp. quadrivalens, Euphorbia flavicoma, Herniaria scabrida y Juniperus phoenicea, en ambas cuadrículas; Achillea odorata, Alyssum montanum, Arctostaphyllos uvaursi, Genista pumila, Geum sylvaticum, Juniperus communis subsp. hemisphaerica, Potentilla cinerea, P. recta, Prunus spinosa, Salsola vermiculata, Sambucus nigra, Satureja intricata y Thymelaea sanamunda, en Ambite; y Atropa baetica, Geranium sanguineum, Helianthemum oelandicum subsp. incanum, Iberis carnosa subsp. granatensis, Laserpitium gallicum, Ononis aragonensis, $O$. fruticosa, O. rotundifolia, O. tridentata, Osyris alba, Phillyrea angustifolia, Pistacia terebinthus, Plantago media, Sanguisorba lateriflora, Sedum dasyphyllum, Silene latifolia, Vicia cracca, V. tenuifolia y Viola rupestris var. arenaria, en Utande.

El tercer paso se deriva del anterior. Consiste en la suma de todos los valores de IOP obtenidos en ese recinto 100C. Se diseña así un segundo índice denominado Índice de Ocupación de la Flora (IOF) que, referido a cada área de estudio, además de las anteriores propiedades contempla también la riqueza florística existente en ese área, pues habrá tantos IOP como plantas se hayan avistado en las cien $1 C$ :

$$
\mathrm{IOF}=\sum_{\mathrm{i}=1}^{\mathrm{n}} \mathrm{IOP}_{\mathrm{i}}
$$

siendo «n» el número de plantas presentes (Riqueza Florística)

Con el IOF, pues, se pueden comparar entre áreas el grado de ocupación florística conjunta, unificando riqueza, frecuencia y abundancia relativa.

\section{Riqueza e IOF comparadas}

Una vez se conocen las plantas avistadas en cada área de estudio y sumados todos sus IOP, se calcularon los dos IOF en cuestión. Además, se calcula la semejanza florística entre ambos territorios según el Índice de Semejanza de Jaccard $(\gamma)$ aplicado por Poulin (2003), cuyos valores oscilan entre 0 (ninguna planta en común) y 1 (todas las plantas son comunes). Así pues, como tercer y último resultado en este artículo, la tabla 1 muestra una síntesis cuantitativa general de riqueza y ocupación. 
TABLA 1

CÓMPUTOS GENERALES DE RIQUEZA Y OCUPACIÓN DE LA FLORA

\begin{tabular}{|c|c|c|c|c|c|c|c|}
\hline \multirow{2}{*}{$\begin{array}{c}\text { TIPOS DE } \\
\text { FITODIVERSIDAD E } \\
\text { ÍNDICES FLORÍSTICOS }\end{array}$} & \multicolumn{2}{|c|}{$\begin{array}{c}\text { 30TWL02 } \\
\text { Utande-100C }\end{array}$} & \multicolumn{2}{|c|}{$\begin{array}{c}\text { 30TVK86 } \\
\text { Ambite-98C }\end{array}$} & \multicolumn{2}{|c|}{$\begin{array}{l}\text { Conjunto } 198 C \\
\text { Utande+Ambite }\end{array}$} & \multirow{2}{*}{$\begin{array}{c}\text { Seme- } \\
\text { janza } \\
\gamma\end{array}$} \\
\hline & $\mathrm{N}$ & $\%$ & $\mathrm{~N}$ & $\%$ & $\mathrm{~N}$ & $\%$ & \\
\hline Plantas (Total)* & 1.023 & $\underline{85,3}$ & 962 & $\underline{80,2}$ & 1.199 & 100,0 & 0,656 \\
\hline Plantas PVR & 275 & 26,9 & 251 & 26,1 & 319 & 26,6 & 0,649 \\
\hline Taxones Autóctonos (Total) & 909 & $\underline{86,3}$ & 847 & $\underline{80,4}$ & 1.053 & 87,8 & 0,668 \\
\hline Taxones Autóctonos PVR & 225 & 24,8 & 198 & 23,4 & 256 & 24,3 & 0,652 \\
\hline Nothotaxones ** & 11 & - & 6 & - & 12 & 1,1 & - \\
\hline ETC (Total) & 989 & $\underline{85,7}$ & 926 & $\underline{80,3}$ & 1.153 & 100,0 & 0,661 \\
\hline ETC (resolución $\left.1 \mathrm{~km}^{2}\right)^{* * *}$ & 294 & 29,7 & 269 & 29,0 & 339 & 29,4 & 0,661 \\
\hline IOF de las ETC (Total) & $2.742,89$ & $\underline{51,6}$ & $2.570,17$ & $\underline{48,4}$ & $5.313,06$ & 100,0 & 一 \\
\hline - IOF de las ETC (res. $1 \mathrm{~km}^{2}$ ) & $1.159,39$ & 42,3 & $1.017,92$ & 39,6 & $2.177,31$ & 41,0 & - \\
\hline
\end{tabular}

Fuente: elaboración propia a partir de Trabajo de Campo (2005-2012).

ABREVIATURAS: PVR, Permanentemente Visibles y Reconocibles. ETC, Entidades Taxonómicas Cartográficas. IOF, Índice de Ocupación de la Flora. N, Número. \%, Porcentajes (aparecen subrayados los referidos al $\mathrm{N}$ del Conjunto Utande+Ambite, dentro de su misma fila; en negrita los que se refieren al $\mathrm{N}$ de Plantas Totales, en su misma columna; y el resto lo hacen respecto al $\mathrm{N}$ total en su mismo apartado y columna). $\gamma$, Índice de Semejanza de Jaccard.

NOTA: Los apartados sombreados implican una modificación metodológica de los datos respecto a los originales.

* Hasta el rango de variedad, se incluyen taxones autóctonos, alóctonos (excepto herbáceos cultivados) e híbridos.

** Salvo Populus x canadensis, todos los híbridos son autóctonos.

*** Se exponen en el Catálogo florístico del Anexo. A las plantas PVR se suman las agrupaciones taxonómicas.

El rango de riqueza detectado oscila entre 950 y 1050 taxones por unidad de $100 \mathrm{~km}^{2}$. Ambite y Utande tienen una riqueza florística diferente pero muy próxima ${ }^{4}$. Sin embargo, Ambite pese a ser menos rica alberga más plantas alóctonas (110 plantas frente a las 104 de Utande); superando esas foráneas el $10 \%$ en ambas áreas.

\footnotetext{
${ }^{4}$ El que en Ambite se hayan prospectado dos cuadrículas menos de las cien establecidas no impide la comparación, pues es bien conocido en Biogeografía que la Curva Especies/Área prácticamente deja ya de aumentar en el tramo próximo a la compleción.
} 
La ligera mayor riqueza de Utande también se expresa en un correlativo mayor valor del IOF. Pero, curiosamente, la abundancia relativa conjunta de toda la flora se deduce como casi idéntica en ambos territorios, pues al dividir el IOF entre el número de ETC se obtienen cocientes técnicamente iguales (2,771 -Utande- $\approx 2,776$-Ambite-).

Las plantas PVR suponen algo más de la cuarta planta de la flora (rango 25-30\%) en ámbitos geográficos característicos de La Alcarria Occidental; mientras que la contrapartida de las plantas No PVR suponen algo menos de las tres cuartas partes de la flora (rango 70-75\%).

Los valores de semejanza indican que existe una muy importante flora común entre Ambite y Utande (786 plantas), al mismo tiempo que una sobrada personalidad florística de cada territorio (237 plantas están en Utande, pero no en Ambite; y 176 en el sentido opuesto). Esas diferencias deben ser atribuidas a que ambas cuadrículas pertenecen a distintos sectores de La Alcarria Occidental. Aunque casi igual, tal semejanza es un poco mayor entre las plantas autóctonas.

\section{CONCLUSIONES}

- La prioridad del método de inventario atribuido al avance en el cubrimiento territorial, a partir de la determinación in situ de las plantas en cualquier época del año, exige diferenciar dos grupos florísticos:

a) Las plantas PVR, cuya distribución y abundancia relativa puede ser bien conocida y comparada a la resolución de la unidad mínima de información $\left(1 \mathrm{~km}^{2}\right)$.

b) Las plantas No PVR, que por presentar problemas de visibilidad fenológica, efimerismo o confusión taxonómica no cumplen la propiedad anterior. Para que la comparación de su distribución sea aceptable, su representación deberá efectuarse con resolución más grosera $\left(4 \mathrm{~km}^{2}\right)$. En este grupo se puede aplicar puntualmente una estrategia operativa de agrupación taxonómica de algunas pocas plantas para aminorar ligeramente aquellas objeciones.

- El rastreo y sistemática aplicados han permitido detectar niveles de riqueza florística muy elevados en unidades de $100 \mathrm{~km}^{2}$, antes no conocidos en La Alcarria Occidental.

- Sin tener en cuenta la composición, los cocientes resultantes de dividir el IOF entre el número de ETC en ambas cuadrículas manifiestan una 
equivalencia en el grado de ocupación de los componentes del manto floral. Esto podría interpretarse como una «identidad» por sus similares respuestas a las pautas paisajísticas de la subregión natural en que se incluyen; pero, también como una prueba de la bondad de los presupuestos metodológicos planteados e índices diseñados.

- Esta metodología permite deslindar con claridad los conceptos biogeográficos de abundancia y frecuencia. El primero hace referencia a la ocupación en términos de cobertura del suelo de un taxón en una unidad territorial bien acotada en forma y superficie. El segundo se refiere a la ocupación en términos de la pura contabilidad de subunidades donde se encuentra presente. En ambos casos es posible la cuantificación. Y tales cuantificaciones, a su vez, pueden servir como criterio para ordenar de mayor a menor las plantas en las respectivas áreas.

- La aplicación de los dos índices (IOP e IOF) da cuenta de manera unificada de la cobertura florística del territorio a escala local. Sus términos aportan concisión a los conceptos biogeográficos a veces discutidos de «abundance», «commonness», «frequency», «occurrence», «occupancy» $\mathrm{y}$ «rareness» empleados en la literatura anglosajona.

- El IOF es bastante realista para las plantas PVR, pero solo una aproximación perfectible para el resto de plantas por objeciones fenológicas, de visibilidad y reconocimiento. Por esta razón, no es posible la ordenación unificada de todo el conjunto floral, sino por separado en cada contingente florístico.

- La detenida lectura en el Anexo del orden por IOP permite una comparación florística muy pormenorizada entre territorios, lo que constituye una novedad en los estudios fitogeográficos españoles.

- La comparación de los IOP planta a planta (o por grupos) y de las distribuciones en cada territorio, unido a la escala de detalle propuesta, aportarán claves para interpretar y explicar aspectos dinámicos, ecológicos, geográficos e históricos sobre la flora. Además, posibilitará también hacer aplicaciones más certeras sobre conservación.

- Por último, queda demostrado cómo el rastreo sistemático del territorio, con el detalle propio de $1 \mathrm{~km}^{2}$, permite la detección de taxones singulares por su rareza. La presencia de tales plantas en los recintos de $100 \mathrm{~km}^{2}$ difícilmente podría haberse constatado de otra manera. Además, el hallazgo de algunas de esas plantas tiene un muy especial interés biogeográfico.

Fecha de recepción: 3 de abril de 2014. Fecha de aceptación: 23 de abril de 2015. 
BiBLIOGRAFÍA

Aizpuru, I.; Aseginolaza, C.; Uribe-Echebarría, P. M.; Urrutia, P. y Zorrakin, I. (2000): Claves ilustradas de la Flora del País Vasco y Territorios Limítrofes. Vitoria, Gobierno Vasco.

Araújo, M. (2003): "The coincidence of people and biodiversity in Europe". Global Ecology and Biogeography, 12, pp. 5-12.

Blondel, J.; Aronson, J.; Bodiou, J.-Y. y Boeuf, G. (2010): The Mediterranean Region. Biological diversity in space and time. New York, Oxford University Press, Second Edition.

Bolòs, O. De y Vigo, J. (1984-2001): Flora dels Països Catalans. Barcelona, Barcino, 4 vols.

Bolòs, O. De; Masalles, R. M.; Vigo, J. y Ninot, J. M. (2005): Flora manual dels Països Catalans. Barcelona, Editorial Pórtic. Tercera edición.

Brown, J. H.; Stevens, G. C. y Kaufman, D. M. (1996): "The geographic range: size, shape, boundaries, and internal structure". Annual Review of Ecology and Systematics, 27, pp. 597-623.

Castroviejo, S. (coord. gen.) (2001): Claves de Flora Ibérica. Vol. I: "Pteridophyta, Gymnospermae, Angiospermae (Lauraceae-Euphorbiaceae)”. Madrid, Real Jardín Botánico, CSIC.

Castroviejo, S. (coord. gen.) (1986-2012): Flora iberica. Plantas vasculares de la Península Ibérica e Islas Baleares 1-8, 10-15, 17-18, 21. Madrid, Real Jardín Botánico, CSIC. Se consultaron, además, en http://www.floraiberica.org borradores y pruebas de imprenta de otros vols. (Fecha de consulta: junio de 2013).

Dale, M. R. T. (1999): Spatial pattern analysis in Plant Ecology. Cambridge, Cambridge Universty Press.

Gabriel, D.; Thies, C. y Tscharntke, T. (2005): "Local diversity of arable weeds increases with landscape complexity". Perspectives in Plant Ecology, Evolution and Systematics, 7, pp. 85-93.

García-Abad, J. J. (2004): "Plantas singulares del tramo norte del valle del Tajuña en la Comunidad de Madrid. Localización en C.U.T.M. de $1 \times 1$ km”, en J. M. Panareda, M. E. Arozena, C. Sanz y N. López (coords.): Estudios en Biogeografía 2004. Terrassa, Aster, pp. 203-214.

García-Abad, J. J. (2006): "El inventario florístico con fines geográficos en C.U.T.M. de $1 \times 1 \mathrm{~km}$. Análisis de la riqueza vascular en La Alcarria Occidental y Mesa de Ocaña”. Serie Geográfica, 13, pp. 117-150.

García-Abad, J. J. (2009): "Geografía de las plantas en La Alcarria Occidental y Mesa de Ocaña (I). Análisis florístico en cinco localidades representativas". Anales de Geografía de la Universidad Complutense, 29/2, pp. 127-153.

García-Abad, J. J.; Gómez, M. y Rodríguez, V. M. (2007): “Atlas geográfico de plantas vivaces de la C.U.T.M. 30TWL02 - Utande (provincia de Guadalajara): Presencia, distribución, abundancia y carácter de los serbales" en S. Gutiérrez y J. J. Sanz Donaire (coords.): Homenaje al Profesor. José Manuel Casas Torres. Madrid, Universidad Complutense de Madrid, pp. 131-144. 
García-Abad, J. J.; Gómez, M. y Rodríguez, V. M. (2009): "Cartografía detallada de plantas vasculares en un sector de la Alta Alcarria, Guadalajara. Utilidad en la detección de enclaves naturales de interés". Lazaroa, 30, pp. 161-174.

Gaston, K. J. y Blackburn, T. M. (2000): Pattern and process in macroecology. Oxford, Blackwell.

Gaston, K. J. y Fuller, R. A. (2009): "The size of species' geographic ranges". Journal of Applied Ecology, 46, pp. 1-9.

Grillo, O. y Gianfranco, V. (eds.) (2011): The dynamical processes of biodiversity -Case studies of evolution and spatial distribution. Rijeka, Croatia, InTech (Open Access Publisher).

Hui, C. y McGeoch, M. A. (2007): "Modeling species distributions by breaking the assumption of self-similarity". Oikos, 116, pp. 2097-2107.

Jiménez-Alfaro, B. (2009): "Evaluación del conocimiento florístico de la Cordillera Cantábrica (España) a partir de bases de datos de biodiversidad". Pirineos, 164, pp. 117-133.

López, N. (2007): "Las plantas vasculares de la Comunidad de Madrid. Catálogo florístico, claves dicotómicas y estudio detallado de la familia 'Compositae' Giseke", Tesis Doctoral, Jardín Botánico de Madrid y Universidad Complutense, Madrid.

Maestre, F. T.; Escudero, A. y Bonet, A. (eds.) (2008): Introducción al análisis espacial de datos en Ecología y Ciencias Ambientales. Madrid, Universidad Rey Juan Carlos.

Mckinney, M. L. (2002): "Do human activities raise species richness? Contrasting patterns in United States plants and fishes". Global Ecology and Biogeography, 11, pp. 343-348.

Meaza, G. (ed.) (2000): Metodología y práctica de la Biogeografía. Barcelona, Editorial del Serbal.

Nichols, W. F; Killingbeck, K. T. y August, P. V. (1998): "The influence of geomorphological heterogeneity on biodiversity II. A landscape perspective". Conservation Biology, 12/2, pp. 371-379.

Nuet, J. y Panareda, J. M. (1991-93): Flora de Montserrat. Barcelona, Publicacions de l'Abadia de Montserrat, 3 vols.

Panareda, J. M. (1996): “Cartografía de la vegetación”. Serie Geográfica, 6, pp. 11-34.

Poulin, R. (2003): "The decay of similarity with geographical distance in parasite communities of vertebrate hosts". Journal of Biogeography, 30, pp. 1609-1615.

Preston, F. W. (1948): "The commonness, and rarity, of species". Ecology, 29, pp. 254283.

Qian, H. y Ricklefs, R. E. (2007): "A latitudinal gradient in large-scale beta diversity for vascular plants in North America". Ecology Letters, 10, pp. 737-744.

Valdés, B.; Talavera, S. y Fernández-Galiano, E. (1987): Flora vascular de Andalucía Occidental. Barcelona, Ketres, 3 vols.

Wilson, P. D. (2008): "The pervasive influence of sampling and methodological artefacts on a macroecological pattern: the abundance-occupancy relationship". Global Ecology and Biogeography, 17, pp. 457-464. 
ANEXO

CATÁLOGO DE TAXONES VASCULARES SILVESTRES Y CULTIVADOS VIVACES AVISTADOS EN LAS CUADRÍCULAS 30TWL02 (UTANDE) Y 30TVK86 (AMBITE) ENTRE 2005 Y 2012

\section{Parte I: Plantas Permanentemente Visibles y Reconocibles y agrupaciones cuya} distribución es representable cartográficamente con resolución de $1 \times 1 \mathrm{~km}$

Los 339 taxones y agrupaciones taxonómicas (considerados ambos como Entidades Taxonómicas Cartográficas o ETC) aquí listados aparecen ordenados por orden alfabético dentro de cada familia, según el sistema taxonómico empleado en «Flora Ibérica» (http//:www.floraiberica.org, Fecha de consulta: junio de 2013).

- Las 224 *ETC antecedidas por un asterisco estaban presentes en las dos cuadrículas de $100 \mathrm{~km}^{2}$. Las 70 ETC sin asterisco y sin subrayar sólo estaban presentes en Utande (Guadalajara, donde se prospectaron las 100 cuadrículas de $1 \times 1 \mathrm{~km}$ ), mientras que las 45 ETC sin asterisco y subrayadas sólo estaban presentes en Ambite (Madrid y Guadalajara, con 98 cuadrículas prospectadas)

- Tras el nombre científico, aparecen unas referencias. Cuando la ETC está en ambas cuadrículas, aparecen en primer lugar las correspondientes a la cuadrícula de Utande y, separadas por un «punto y coma», las de Ambite en segundo lugar. Si, estando en ambas cuadrículas, se pone una sola referencia, se entiende que se aplica por igual a las dos.

- La primera referencia aparece entre paréntesis y corresponde a caracteres de la planta (Alóctonas: ad, adventicia; c, cultivada; i, alóctona invasora; n, naturalizada no invasora; s, asilvestrada de cultivo; Autóctonas: a, autóctona; Protegidas, con figura de amenaza: ep, en peligro; v, vulnerable; sah, sensible a la alteración del hábitat (sólo Comunidad de Madrid); ie, de interés especial; Endemismos Ibérico-Baleares: e). Si tiene varios caracteres, aparecen separados por comas. Si no aparece referencia entre paréntesis, es autóctona. Las plantas protegidas son autóctonas, salvo que se ponga otro carácter. Se emplea el símbolo interrogación (i?) cuando se tienen dudas sobre el carácter.

- La segunda referencia, separada por un espacio de la primera, corresponde a tres valores numéricos que indican por orden lo siguiente: Valor del IOP en la cuadrícula/Número de orden según IOP en su grupo florístico/Frecuencia (número de cuadrículas $1 \times 1 \mathrm{~km}$ donde estaba presente). 
- Las agrupaciones taxonómicas se ponen en negrita. Bajo ellas y con sangría se refieren los taxones cuya presencia se ha podido reconocer (se aplican igual los asteriscos y subrayados o no).

NOTAS: Se considera como «invasora» la planta espontánea así contemplada en Sanz et al. (2004). Se pone la abreviatura «cf» (confert) después del nomen genérico, en los casos de determinación específica probable, pero no confirmada.

\section{Aceraceae}

Acer campestre L. (c) 0,01/254/1

*Acer monspessulanum L. (ie) 1,75/154/28; (no es «ie» en Madrid) 0,57/174/12

*Acer negundo L. (c) 0,13/241/4; 0,01/254/1 Acer platanoides L. (c) 0,02/275

Acer pseudoplatanus L. (c) $0,01 / 281 / 1$

Agavaceae

Agave spp. (c) $0,02 / 243 / 2$

Agave cf americana L. (c)

Yucca aloifolia L. (c) 0,11/214/2

Anacardiaceae

${ }^{*}$ Pistacia terebinthus L. 0,26/216/8; $4,17 / 88 / 48$

*Rhus coriaria L. (n) 0,41/203/5; 0,80/156/8

\section{Apocynaceae}

*Vinca spp. (cs) 0,2/226/2; 0,41/178/5

*Vinca difformis Pourr. (c)

Vinca major L. (cs)

\section{Araceae}

*Hedera helix L. (a,c,s) 2,86/127/28; $1,13 / 139 / 14$

\section{Aspleniaceae}

*Asplenium trichomanes subsp. quadrivalens D.E. Meyer 0,11/243/2; 0,1/218/1

${ }^{*}$ Ceterach officinarum Willd. subsp. officinarum 3,62/112/38; 2,13/115/33

\section{Berberidaceae}

Mahonia aquifolium (Pursh) Nutt. (s) $0,01 / 254 / 1$

\section{Betulaceae}

Alnus glutinosa (L.) Gaertn. (ie) 0,41/203/5

*Corylus avellana L. (c, ie) 0,16/237/7; (c) $0,14 / 207 / 5$

\section{Boraginaceae}

*Lithodora fruticosa (L.) Griseb. 9,95/33/80; $9,34 / 41 / 88$

\section{Campanulaceae}

Jasione sessiliflora Boiss. \& Reut. 0,1/251/1

Cannabaceae

${ }^{*}$ Humulus lupulus L. 1,55/157/11;

2,65/104/16

Caprifoliaceae

*Lonicera etrusca Santi 10,96/26/91; $10,27 / 34 / 90$

Lonicera implexa Aiton var. implexa

$1,84 / 125 / 22$

*Lonicera japonica Thunb. (s) 0,03/270/3; $0,21 / 193 / 3$

*Lonicera periclymenum subsp. hispanica (Boiss. \& Reuter) Rivas Goday 1,5/158/15; 0,82/153/10

Lonicera xylosteum L. 0,01/281/1

*Sambucus ebulus L. 2,5/134/25; 0,7/165/7 
*Sambucus nigra L. 1,87/151/28; (ie) Cistus ladanifer L. subsp. ladanifer $0,13 / 208 / 4$

2,25/142/10

Viburnum lantana L. 1,59/156/24

Cistus laurifolius L. 5,77/83/55

Cistus monspeliensis L. (c,s) 0,10/218/1

Caryophyllaceae

Arenaria cavanillesiana (Font Quer \& Rivas Goday) Nieto Fel. (e) 1,44/133/18

*Dianthus pungens L. 5,10/91/51; 2,59/105/43

Dianthus pungens subsp. brachyanthus (Boiss.) Bernal, Fernández Casas, G.López, Laínz \& Muñoz Garm.

${ }^{*}$ Dianthus pungens subsp. hispanicus (Asso) O. Bolòs \& Vigo (e)

Gypsophila bermejoi G. López (ie,e) 0,21/193/3

*Gypsophila struthium L. subsp. struthium (e) $0,22 / 222 / 4 ; 0,31 / 187 / 4$

*Herniaria scabrida Boiss. 0,01/281/1; 0,12/212/3

Paronychia argentea Lam. var. argentea 0,8/188/8

*Paronychia capitata var. capitata 3,42/120/36; 4,4/85/53

*Cistus salviifolius L. 0,10/251/1; 0,2/199/2

*Fumana ericifolia Wallr. 10,84/27/88; $10,93 / 27 / 88$

*Fumana procumbens (Dunal) Gren. \& Godr. 6,18/75/69; 3,49/94/43

*Fumana thymifolia (L.) Spach ex Webb $6,41 / 71 / 65 ; 8,46 / 48 / 81$

Halimium umbellatum subsp. viscosum (Willk.) O. Bolòs \& Vigo 1,01/176/11

*Helianthemum

9,3/39/93; 9,46/39/88

*Helianthemum apenninum (L.) Mill. subsp. apenninum

*Helianthemum apenninum subsp. stoechadifolium (Brot.) Samp.

*Helianthemum asperum Lag. ex Dunal subsp. asperum (e)

*Helianthemum cinereum subsp. rotundifolium (Dunal) Greuter \& Burdet $17,61 / 7 / 93 ; 23,2 / 3 / 98$

* Silene latifolia Poir. 0,02/275/2; $1,16 / 137 / 17$

Silene legionensis Lag. (e) 2,31/140/24

* Silene mellifera Boiss. \& Reut. (e) 6,73/62/70; 7,16/61/77

*Telephium imperati L. subsp. imperati $1,02 / 175 / 21 ; 0,79 / 158 / 16$

Chenopodiaceae

Atriplex halimus L. (c) 0,02/243/2

*Helianthemum hirtum (L.) Mill. subsp. hirtum 9,56/37/89; 13,60/15/97

Helianthemum oelandicum subsp. incanum (Willk.) G. López 0,81/186/9

Helianthemum squamatum (L.) Dum. Cours. $1,85 / 123 / 8$

Helianthemum syriacum (Jacq.) Dum. Cours. 0,21/193/3

\section{Compositae}

*Bassia prostrata (L.) G. Beck 0,20/226/2; 2,07/117/27

Salsola vermiculata L. 0,20/199/2

Achillea ageratum L. 0,53/198/8

Achillea millefolium L. 4,10/106/50

*Achillea odorata L. 7,02/60/72; 0,1/218/1

Artemisia absinthium L. 1,93/150/22

*Artemisia campestris L. subsp. campestris

Cistaceae

${ }^{*}$ Cistus albidus L. 4,47/102/45; 10,79/29/77 2,33/138/29; 0,02/243/2

Cistus clusii Dunal subsp. clusii (c) *Artemisia herba-alba Asso 0,03/270/3; $0,01 / 254 / 1$ $0,4 / 180 / 4$ 
*Atractylis humilis L.

0,93/180/12; 7,53/55/75

*Carduncellus monspelliensium

All.

2,43/137/27; 0,93/149/12

*Carlina corymbosa subsp. hispanica (Lam.)

O. Bolòs \& Vigo 10,42/29/97; 11,76/23/96

Carlina vulgaris subsp. spinosa (Velen.)

Vandas 4,97/93/56

*Centaurea aspera L. subsp. aspera 7,86/53/84; 5,96/72/65

*Centaurea aspera L. subsp. aspera

Centaurea aspera L. («subinermis»)

*Centaurea ornata Willd. (s. srt.)

4,5/100/45; 8,1/51/81

Cynara scolymus L. (s) 0,1/218/1

${ }^{*}$ Dittrichia viscosa (L.) Greuter (ad)

0,01/281/1; 0,11/214/2

${ }^{*}$ Helichrysum serotinum Boiss. 0,56/196/11; 0,23/192/5

*Helichrysum stoechas (L.) Moench subsp. stoechas 10,13/31/92; 12,65/19/95

Hieracium spp. 0,6/194/6

Hieracium cf loscosianum Scheele

*Jasonia saxatilis (Lam.) Guss. 2,62/131/28;

$1,11 / 141 / 12$

Jurinea humilis (Desf.) DC. 0,87/151/15

*Klasea pinnatifida (Cav.) Cass. 0,1/251/1; $11,71 / 24 / 94$

Launaea fragilis (Asso) Pau 0,61/169/7

Phagnalon rupestre (L.) DC. 1,01/145/11

*Pilosella spp. 6,7/63/67; 2,4/109/24

*Pilosella pseudopilosella (Ten.) Soják

*Pilosella tardans (Peter) Soják

*Santolina chamaecyparissus

10,41/30/96; 6,67/66/73

*Staehelina dubia L. 11,39/24/86; 14,48/10/95

\section{Cornaceae}

Cornus sanguinea L. 3,5/117/38

\section{Crassulaceae}

Sedum acre L. 2,1/147/21
*Sedum album L. 6,23/74/65; 5,73/73/60

Sedum amplexicaule DC. subsp. amplexi-

caule 0,82/153/10

Sedum dasyphyllum L. subsp. dasyphyllum $0,40 / 208 / 4$

Sedum gypsicola Boiss. \& Reut.

$0,1 / 218 / 1$

*Sedum sediforme (Jacq.) Pau 5,68/86/64;

$7,53 / 55 / 72$

*Umbilicus rupestris (Salisb.) Dandy

1/177/10; 2,48/107/32

(e) Cruciferae

Alyssum montanum L. 0,4/180/4

*Alyssum serpyllifolium Desf. 6,25/73/67;

6,35/68/71

Arabis hirsuta (L.) Scop. 3,63/111/48

*Arabis planisiliqua (Pers.) Rchb. 4,73/97/50; 3,07/98/37

*Biscutella valentina (Loefl. ex L.) Heywood var. valentina 1,85/153/23; 4,99/78/58

Brassica repanda subsp. confusa (Emb. \& Maire) Heywood 0,1/218/1

Brassica repanda subsp. gypsicola Gómez Campo (e) 0,11/243/2

*Hormatophylla lapeyrousiana (Jord.) P. Küpfer 5,41/88/55; 1,02/144/12

Iberis carnosa subsp. granatensis (Boiss. \& Reut.) Moreno (e) 0,41/203/5

*Lepidium hirtum (L.) Sm. 3,45/119/39; 0,95/148/14

Lepidium subulatum L. 0,21/193/3

*Matthiola fruticulosa (Loefl. ex L.) Maire

L. subsp. fruticulosa 7,32/56/75; 2,94/101/33

\section{Cupressaceae}

*Cupressus arizonica Greene (c) 0,81/186/9; $1,05 / 142 / 15$

*Cupressus sempervirens L. (c) 0,01/281/1; (c,s) 0,91/150/10

*Juniperus communis L. subsp. communis 0,92/181/20;0,01/254/1 
* Juniperus communis subsp. hemisphaerica (K. Presl) Nyman 2,46/135/48; 0,03/239/3

*Juniperus oxycedrus L. 3,28/121/52; $0,1 / 218 / 10$

Juniperus oxycedrus subsp. badia ( $\mathrm{H}$. Gay) Debeaux

*Junperus oxycedrus subsp. oxycedrus

*Juniperus phoenicea L. 0,09/264/9;

$0,01 / 254 / 1$

*Juniperus thurifera L. (ie) $0,18 / 236 / 18$; (sah,a,s) 0,02/243/2

\section{Cyperaceae}

Carex cuprina (I. Sándor ex Heuff.) Nendtv. ex A. Kern. 0,9/182/9

*Carex distachya/hallerana 12,08/20/89; 13,46/16/95

*Carex distachya Desf.

${ }^{*}$ Carex hallerana Asso

Carex flacca Schreb. 5,85/81/57

*Carex hordeistichos Vill. 0,10/251/1; $0,10 / 218 / 1$

Carex paniculata L. subsp. paniculata 0,1/251/1

Carex riparia Curtis 0,2/226/2

Cyperus longus L. 1,1/173/11

Schoenoplectus lacustris subsp. glaucus (Sm. ex Hartm.) Bech. 0,21/224/3

Schoenus nigricans L. 1,34/166/17

*Scirpioides holoschoenus (L.) Sojàk 8,58/46/84; 4,93/79/55

\section{Dipsacaceae}

*Cephalaria leucantha (L.) Roem. \& Schult. 12,25/19/91; 14,04/14/96

* Scabiosa columbaria L. 6,30/72/63; $2,31 / 111 / 24$

\section{Eleagnaceae}

*Eleagnus angustifolia L. (c) 0,01/281/1; $0,01 / 254 / 1$

\section{Ephedraceae}

*Ephedra distachya/nebrodensis 0,10/251/

1; 1,93/120/22

Ephedra distachya L. subsp. distachya (ie, en Castilla-La Mancha)

*Ephedra nebrodensis Tineo ex Guss. subsp. nebrodensis (ie, en C-LM)

Ephedra fragilis Desf. subsp. fragilis (ie) $0,71 / 163 / 8$

\section{Equisetaceae}

*Equisetum ramosissimum L. 3,52/115/37; $2,25 / 113 / 21$

\section{Ericaceae}

*Arctostaphyllos uva-ursi (L.) Spreng. 3,05/ 124/25; 0,15/205/6

Erica scoparia L. subsp. scoparia 0,01/281/1

\section{Euphorbiaceae}

* Euphorbia flavicoma DC. subsp. flavicoma 0,1/251/1;0,71/163/8

*Euphorbia minuta Loscos \& J. Pardo (e) 4,71/98/48; 0,82/153/10

${ }^{*}$ Euphorbia nicaeensis All. var. nicaeensis 12,65/14/95; 10,48/32/91

* Mercurialis tomentosa L. 3,87/108/45; $3,77 / 92 / 44$

\section{Fagaceae}

*Quercus coccifera L. 3,75/109/32; 17,56/6/98 *Quercus faginea Lam. subsp. faginea $19,06 / 3 / 97 ; 8,60 / 46 / 70$

*Quercus ilex subsp. ballota (Desf.) Samp. 18,53/6/98; 24,70/1/98

*Quercus x auzandrii Gren. \& Godr. $0,01 / 281 / 1 ; 0,02 / 243 / 2$

*Quercus $\mathrm{x}$ senneniana A. Camus $0,03 / 270 / 3 ; 0,01 / 254 / 1$

\section{Geraniaceae}

Geranium sanguineum L. 0,3/211/3 


\section{Globulariaceae}

Globularia alypum L. 0,01/254/1

*Globularia vulgaris L. 9,73/35/85; 6,1/71/64

\section{Gramineae}

*Arundo donax L. (c) 0,21/224/3; (c,i) 2,4/109/24

*Brachypodium phoenicoides (L.) Roem. \& Schult. 11,4/23/99; 10,7/30/92

*Brachypodium retusum (Pers.) P. Beauv. 33,55/1/98; 4,3/86/43

*Brachypodium sylvaticum (Huds.) P. Beauv. 4,45/103/49; 0,41/178/5

*Dactylis glomerata L. 9,2/41/92; 6,75/65/66

${ }^{*}$ Dactylis hispanica Roth subsp. hispanica 14,5/12/97; 15,85/8/98

Koeleria castellana Boiss. \& Reut. (e) $1,61 / 132 / 8$

*Koeleria vallesiana (Honck.) Gaudin $15,8 / 10 / 95 ; 12,15 / 22 / 84$

* Melica minuta L. 0,2/226/2; 1,73/127/20

Molinia caerulea subsp. arundinacea (Schrank) Soják 2,7/129/27

*Phalaris arundinacea L. 4,93/94/52; $0,3 / 189 / 3$

Phalaris coerulescens Desf. (ad) 0,1/251/1

*Phragmites australis (Cav.) Trin. ex Steud. 6,43/70/55; 3,91/91/31

Phyllostachys viridiglaucescens (Carrière)

Rivière \& C. Rivière (c) 0,11/243/2

Piptatherum miliaceum (L.) Coss. subsp. miliaceum (ad) 0,11/214/2

*Piptatherum paradoxum (L.) P. Beauv.

1,36/164/19; 4,72/81/52

*Saccharum ravennae (L.) Murray (c) $0,1 / 251 / 1 ; 0,1 / 218 / 1$

*Stipa sect. Leiostipa 6,65/65/62; 8,36/49/74 * Stipa capillata L.

*Stipa lagascae Roem. \& Schult.

* Stipa offneri Breistr.

*Stipa sect. Stipa 6,8/61/71; 6,26/70/62

*Stipa iberica Martinovský
Stipa pauneroana (Martinovský) Vázq.

Pardo \& Devesa

*Stipa tenacissima L. 0,27/215/9; 9,88/35/85

\section{Hippocastanaceae}

Aesculus hippocastanum L. (c) 0,04/267/4

\section{Hydrangeaceae}

Philadelphus pubescens Loisel. (c) 0,1/251/1

\section{Hypolepidaceae}

Pteridium aquilinum (L.) Kuhn subsp. aquilinum (n) 0,1/218/1 (comunicación personal J. M. Martínez Labarga, 2009)

\section{Juglandaceae}

*Juglans regia L. (c,s) 3,98/107/68; 2,56/106/49

\section{Juncaceae}

Juncus acutiflorus Ehrh. ex Hoffmanns. 0,1/251/1

*Juncus inflexus L. subsp. inflexus 4,8/96/48; $0,6 / 171 / 6$

\section{Labiatae}

${ }^{*}$ Hyssopus officinalis L. 1,86/152/24; 0,96/147/15

*Lavandula latifolia (L.) Medik. 18,87/4/93; $12,71 / 18 / 89$

Lavandula pedunculata (Miller) Cav. $1,31 / 169 / 14$

*Marrubium supinum L. 6/79/69; 3,22/97/43 *Marrubium supinum $\mathrm{x}$ M. vulgare 0,04/267/4; 0,04/238/4

*Marrubium vulgare L. 5,22/90/72; 5,08/77/67

*Phlomis herba-venti L. 8,83/44/91; $6,64 / 67 / 79$

*Phlomis lychnitis L. 10,8/28/99; 12,95/17/98

${ }^{*}$ Rosmarinus officinalis L. 14,03/13/64; $15,23 / 9 / 78$

* Salvia lavandulifolia Vahl subsp. lavandulifolia (e) 17,58/8/86; 9,57/38/84

Salvia officinalis L. (s) 0,1/218/1 
*Salvia verbenaca L. 9,6/ 36/96; 8,85/44/87

*Satureja intricata Lange (e) 9,47/38/80; $0,6 / 171 / 6$

*Sideritis hirsuta L. 9,01/43/91; 7,93/53/91

* Sideritis incana L. 8,71/45/82; 5,36/75/59

*Teucrium capitatum L. subsp. capitatum $7,6 / 54 / 85 ; 7,71 / 54 / 81$

*Teucrium chamaedrys L. 14,71/11/93; $12,26 / 21 / 95$

Teucrium fruticans L. (c) 0,01/254/1

*Teucrium gnaphalodes L Hér. (e) 9,3/39/90; $9,81 / 36 / 93$

Thymus lacaitae Pau (e) 1/146/10

*Thymus mastichina L. subsp. mastichina (e) $2,44 / 136 / 28 ; 0,1 / 218 / 1$

*Thymus vulgaris L. subsp. vulgaris $17,31 / 9 / 82 ; 24,21 / 2 / 97$

Thymus x brachychaetus (Willk.) Cout. $0,52 / 199 / 7$

*Thymus zygis subsp. sylvestris (Hoffmanns. \& Link) Brot. ex Cout. (e) 18,85/5/98; 18,4/4/92

\section{Lauraceae}

*Laurus nobilis L. (c) 0,14/238/5; 0,03/239/3

\section{Leguminosae}

*Argyrolobium zanonii (Turra) P.W. Ball subsp. zanonii 7,93/52/82; 8,76/45/90

Astragalus glycyphyllos L. 0,32/209/5

Astragalus granatensis Lam. (ie) 0,71/190/8

*Astragalus incanus L. subsp. incanus 6,02/77/68 4,54/84/55

*Astragalus monspessulanus subsp. gypsophilus Rouy 2,91/126/30; 2,78/102/29

Cercis siliquastrum L. (c) 0,20/199/2

*Colutea hispanica Talavera \& Arista (ie,e) 2,23/143/34; (no es «ie» en Madrid) $4,63 / 83 / 52$

*Coronilla glauca L. (ie) 0,2/226/2; (no es «ie» en Madrid) 0,21/193/3

*Coronilla minima subsp. lotoides (W.D.J. Koch) Nyman 6,55/68/61; 2,24/114/26
*Coronilla minima L. subsp. minima 6,6/66/69; 0,46/177/10

Cytisus scoparius (L.) Link subsp. scoparius $0,22 / 222 / 4$

*Dorycnium pentaphyllum Scop. 8,02/51/82; $8,22 / 50 / 75$

* Genista pumila (Viehr.) Talavera \& L. Sáez (e) $1,7 / 155 / 17 ; 0,2 / 199 / 2$

*Genista scorpius (L.) DC. 28,67/2/99; $18,4 / 4 / 96$

* Gleditschia triacanthos L. (c) $0,01 / 281 / 1$; $0,03 / 239 / 3$

*Hedysarum boveanum subsp. europaeum Guitt. \& Kergélen 8,53/47/67; 1,94/119/23

*Hippocrepis commutata Pau (e) 7,54/55/79; 9,23/43/89

*Medicago suffruticosa Ramond ex DC. 6,66/64/72; 3,35/95/41

*Onobrychis saxatilis (L.) Lam.

2,33/138/26; 0,1/218/1

Ononis aragonensis Asso 0,2/226/2

Ononis fruticosa L. 0,31/210/4

*Ononis natrix L. 1,32/168/15; 0,5/175/5

Ononis rotundifolia L. 0,11/243/2

*Ononis tridentata L. subsp. tridentata 0,41/203/5; 1,12/140/13

*Retama sphaerocarpa (L.) Boiss. 5,49/87/60; 3,96/89/63

*Robinia pseudoacacia L. (c,i) 0,6/194/15; $1,87 / 121 / 25$

*Sophora japonica L. (c,s) 0,14/238/5; (c) $0,01 / 254 / 1$

*Spartium junceum L. (c) 0,11/243/2; (c,s) $0,11 / 214 / 2$

Vicia sect. Cracca 0,9/182/9

Vicia cracca L.

Vicia tenuifolia Roth.

\section{Liliaceae}

*Aphyllanthes monspeliensis L. 9,99/32/87; $10,87 / 28 / 93$ 
*Asparagus acutifolius L. 3,7/110/46; $10,6 / 31 / 97$

${ }^{*}$ Ruscus aculeatus L. 0,1/251/1; 0,72/161/9

\section{Linaceae}

Linum campanulatum L. (ie) 0,2/226/2

${ }^{*}$ Linum suffruticosum L. 12,56/17/92; $9,4 / 40 / 79$

\section{Malvaceae}

*Alcea rosea L. (s) 0,7/191/7; 0,1/218/1

*Althaea officinalis L. 0,7/191/7; 0,8/156/8

Lavatera triloba L. var. triloba $0,63 / 166 / 9$

\section{Meliaceae}

Melia azedarach L. (c) 0,01/254/1

\section{Moraceae}

${ }^{*}$ Ficus carica L. (c,n,s) 2,08/149/46; 3,77/92/62

${ }^{*}$ Morus spp. (c) 0,23/219/5; 0,35/184/8

*Morus alba L.

*Morus nigra L.

\section{Nyctaginaceae}

Mirabilis jalapa L. (c) 0,01/281/1

\section{Oleaceae}

*Fraxinus angustifolia Vahl 2,13/144/24; 0,84/152/12

Fraxinus excelsior L. ( $a$ a?,vu) 0,11/243/2

${ }^{*}$ Fraxinus oxycarpa Willd. 0,01/281/1; $0,02 / 243 / 2$

*Jasminum fruticans L. 5,91/80/63; $14,32 / 12 / 97$

Jasminum officinale L. (c) 0,2/226/2

Ligustrum lucidum W.T. Aiton $0,02 / 275 / 2$

*Ligustrum vulgare L. (a) 5,05/92/52; (a,c) $0,21 / 193 / 3$

*Olea europaea L. (c,s) 9,04/42/64; $16,54 / 7 / 83$
*Phillyrea angustifolia L. 0,02/275/2; 0,15/205/6

*Syringa vulgaris L. (c,s) 0,26/216/8; (c) $0,27 / 191 / 9$

\section{Pinaceae}

Cedrus atlantica (Endl.) Carrière (c) $0,32 / 185 / 5$

*Pinus halepensis Mill. (c,s) 4,58/99/30; (c,s,a) 4,66/82/52

*Pinus nigra J.F. Arnold (c,s) 1,14/172/15; $0,03 / 239 / 3$

*Pinus pinaster Aiton (c,s) 0,06/265/6; (c) $0,02 / 243 / 2$

*Pinus pinea L. (c) 0,01/281/1; 0,07/236/7

*Pinus sylvestris L. (c) $0,11 / 243 / 2$; $0,02 / 243 / 2$

\section{Plantaginaceae}

*Plantago albicans L. 3,1/123/31; 7,5/57/75

Plantago holosteum Scop. 1,4/161/14

*Plantago lanceolata L. 11,35/25/100; 9,3/42/90

*Plantago major L. 3,5/117/35; 1,2/136/12 Plantago maritima subsp. serpentina (All.) Arcang. 2,11/145/22

Plantago media L. 0,11/243/2

*Plantago sempervirens Crantz 3,62/112/47; $6,98 / 64 / 74$

\section{Platanaceae}

Platanus hispanica Mill. ex Münchh. (c) $0,01 / 254 / 1$

Platanus orientalis L. (c) $0,1 / 251 / 1$

\section{Plumbaginaceae}

(c) Armeria alliacea subsp. matritensis (Pau) Borja, Rivas Goday \& Rivas Mart. (e) $1,73 / 127 / 20$

*Plumbago europaea L. 0,9/182/18; $1,43 / 134 / 26$ 


\section{Polygonaceae}

${ }^{*}$ Rumex crispus L. 4,5/100/45; 4,2/87/ 42

Rumex induratus Boiss. \& Reut. 0,01/281/1

*Rumex pulcher subsp. woodsii (De Not.) Arcang. 8,3/50/83; 7,1/62/80

\section{Primulaceae}

*Coris monspeliensis L. 8,41/49/85; $6,31 / 69 / 67$

\section{Punicaceae}

*Punica granatum L. (c) $0,03 / 270 / 3$; (c,s) $0,62 / 168 / 8$

\section{Ranunculaceae}

*Ranunculus bulbosus L. 2,1/147/21; 0,13/208/4

*Ranunculus bulbosus subsp. aleae (Willk.) Rouy \& Foucaud

${ }^{*}$ Ranunculus bulbosus subsp. bulbosus

Thalictrum minus subsp. pubescens Schleich. ex. Arcang. 1,5/158/15

\section{Resedaceae}

Sesamoides purpurascens (L.) G. López $0,3 / 211 / 3$

\section{Rhamnaceae}

*Rhamnus alaternus L. 4,85/95/56; $11,43 / 25 / 93$

*Rhamnus lycioides L. 0,54/197/9; $7,39 / 58 / 82$

*Rhamnus infectoria L. 5,83/82/70; 2,02/118/31

\section{Rosaceae}

*Agrimonia eupatoria L. subsp. eupatoria 5,71/85/67; 1,71/129/27

Amelanchier ovalis Medik. 2,62/131/28

*Cotoneaster tomentosus (Aiton) Lindl. (v) 0,43/202/7; 0,01/254/1

*Crataegus monogyna Jacq. 12,6/16/99;
$12,5 / 20 / 97$

${ }^{*}$ Cydonia oblonga Mill. (c,s) 0,7/191/16; $1,86 / 122 / 33$

Eriobotrya japonica (Thunb.) Lindl. (c) $0,1 / 218 / 1$

${ }^{*}$ Geum sylvaticum Pourr. 1,3/171/13; $0,2 / 199 / 2$

*Malus domestica (Borkh.) Borkh. (c,s) 1,39/163/22; 0,75/160/21

Mespilus germanica L. (c) $0,01 / 254 / 1$ Potentilla cinerea Chaix ex Vill. 0,4/180/4

*Potentilla neumanniana Rchb. 5,73/84/60; $1,05 / 142 / 15$

Potentilla recta L. 0,1/218/1

${ }^{*}$ Potentilla reptans L. 7,3/57/73; 4,86/80/48

*Prunus armeniaca L. (c,s) 0,25/218/7; 0,63/166/9

*Prunus avium L. (c,n,s,ie) 2,11/145/40; (c,s) 0,79/158/16

*Prunus cerasifera Ehrh. (c) 0,02/275/2; (c,s) 0,13/208/4

*Prunus cerasus L. (c,s) 0,84/185/12; $0,13 / 208 / 4$

*Prunus domestica L. (c,s) 2,69/130/44; $1,62 / 131 / 27$

*Prunus dulcis (Mill.) D.A. Webb (c,s) 4,28/105/59; 10,37/33/89

Prunus mahaleb L. (ie) 0,23/219/5

*Prunus persica (L.) Batsch (c) 0,04/267/4; $0,02 / 243 / 2$

*Prunus spinosa L. 7,06/59/76; 0,31/187/4

Pyracantha rogersiana (A.B.Jack.) Chitt. (c) $0,01 / 254 / 1$

*Pyrus communis L. (c,s) 1,33/167/25; $1,21 / 135 / 22$

*Pyrus cordata Desv. (c,s) 0,05/266/5; (c) $0,02 / 243 / 2$

${ }^{*}$ Rosa spp. 12,61/15/100; 7,06/63/91

${ }^{*}$ Rosa agrestis

${ }^{*}$ Rosa canina («sensu lato»)

Rosa gallica (s)

*Rosa micrantha 
Rosa moschata (s)

* Rosa pouzinii

${ }^{*}$ Rubus caesius L. 2,55/133/24; 1,85/123/17

*Rubus ulmifolius Schott 12,55/18/100;

$7,18 / 60 / 73$

Sanguisorba lateriflora (Coss.) A. Braun \& C.D. Bouché 0,41/203/5

*Sanguisorba minor/rupicola/verrucosa 9,8/34/98; 9,75/37/81

*Sanguisorba minor subsp. balearica

(Bourg. ex Nyman) Muñoz Garm. \& C.

Navarro

*Sanguisorba minor L. subsp. minor

Sanguisorba rupicola (Boiss. \& Reut.) A.

Braun \& C.D. Bouché

*Sanguisorba verrucosa (Link ex G. Don)

Ces.

Sorbus aria (L.) Crantz (ie) 0,12/242/3

*Sorbus domestica L. (c,n,ie) 0,3/211/12; (c) $0,12 / 212 / 3$

Sorbus torminalis (L.) Crantz (ie,sah) $0,02 / 275 / 2$

\section{Rubiaceae}

${ }^{*}$ Galium lucidum All. subsp. lucidum 8,52 48877,325978

${ }^{*}$ Rubia peregrina L. $11,82 / 22 / 87$; 14,22/13/96

*Rubia tinctorum L. (n) $1,5 / 158 / 15$; 2,31/111/24

\section{Rutaceae}

${ }^{*}$ Ruta angustifolia Pers. 3,21/122/33; 3,94/90/40

*Ruta montana L. 4,32/104/54; 5,73/73/69

\section{Salicaceae}

${ }^{*}$ Populus alba L. 2,71/128/31; 1,14/138/15

*Populus nigra L. (c,n,s) 6,16/76/61; 3,03/99/36

Populus simonii Carrière 0,1/218/1

${ }^{*}$ Populus $\mathrm{x}$ canadensis Moench. (c,s)
3,52/115/33; 0,36/183/9

*Salix babilonica L. (c) $0,14 / 238 / 5$;

$0,32 / 185 / 5$

*Salix purpurea L. 1,31/169/17; 0,72/161/9

Santalaceae

*Osyris alba L. 0,52/199/7; 2,47/108/22

\section{Scrophulariaceae}

*Digitalis obscura L. subsp. obscura 6,57/67/69; 3,25/96/37

* Scrophularia canina L. var. canina 0,94/179/22;0,61/169/16

\section{Simaroubaceae}

*Ailanthus altissima (Mill.) Swingle (c,i,s) 0,99/178/18; 2,69/103/35

\section{Solanaceae}

Atropa baetica Willk. (ep) 0,01/281/1

Lycium europaeum L. (c,s) 0,23/219/5

*Solanum dulcamara L. 2,31/140/24; $2,11 / 116 / 22$

\section{Sparganiaceae}

Sparganium erectum L. subsp. erectum $0,2 / 226 / 2$

\section{Tamaricaceae}

*Tamarix spp. 0,75/189/12; 0,06/237/6

*Tamarix gallica L.

Thymelaeaceae

*Daphne gnidium L. 3,57/114/36;

$8,06 / 52 / 83$

*Thymelaea pubescens (L.) Meisn.

7,28/58/80; 1,75/126/31

Thymelaea sanamunda All. 0,3/189/3

\section{Tiliaceae}

Tilia platyphyllos Scop. subsp. platyphyllos (c) $0,03 / 270 / 3$ 
Typhaceae

*Typha angustifolia/T. dominguensis

0,5/201/5; 0,10/218/1

*Typha domingensis Pers.

*Typha latifolia L. 1,4/161/14; 0,5/175/5

\section{Ulmaceae}

Celtis australis L. ( $i a$ ?) 0,02/243/2 (una de las dos cuadrículas: comunicación personal J. M. Martínez Labarga, 2009)

*Ulmus minor Mill. (a,c) 5,24/89/53; $3,01 / 100 / 31$

*Ulmus pumila L. (c,s) 1,1/173/20; $1,64 / 130 / 20$

\section{Umbelliferae}

*Bupleurum fruticescens L. subsp. fruticescens 1,35/165/18; 14,41/11/94

Bupleurum fruticosum L. (c,s) 0,2/199/2
*Eryngium campestre L. 11,95/21/100; $11,3 / 26 / 98$

${ }^{*}$ Foeniculum vulgare Mill. 6,01/78/61; $5,33 / 76 / 56$

Laserpitium gallicum L. subsp. gallicum $0,2 / 226 / 2$

\section{Violaceae}

Viola rupestris var. arenaria (DC.) G. Beck $0,3 / 214 / 3$

*Viola sect. Viola 6,5/69/65; 0,6/171/6

*Viola alba subsp. dehnhardtii (Ten.) W.

Becker

Viola odorata L. (n)

\section{Vitaceae}

*Vitis vinifera L. (c,s) 3,02/125 41; $8,49 / 47 / 69$

Referencia Bibliográfica: Sanz, M.; Dana, E. y Sobrino, E. (2004): Atlas de las plantas alóctonas invasoras de España. Madrid, Ministerio de Medio Ambiente.

\section{RESUMEN}

Con objeto de precisar conceptos biogeográficos y conocer la riqueza florística detallada, se presenta un método de inventario a escala local que emplea la cuadrícula U.T.M. de $1 \times 1 \mathrm{~km}$ (ED50) como unidad de información básica y la de 10×10 km como territorio de estudio. Se eligen dos cuadrículas pertenecientes a la misma subregión natural (La Alcarria Occidental), pero en sectores diferentes (30TWL02 y 30TVK86), para propiciar su comparación. Los resultados han sido: a) diferenciación de dos contingentes florísticos por razones fenológicas, b) diseño y formulación dos nuevos índices que cuantifican la ocupación de la flora, c) detección de unos altos valores de riqueza (en torno a mil taxones en $100 \mathrm{~km}^{2}$ ); y d) presentación de un Catálogo de plantas cuya distribución es representable con resolución de 1 km². La comparación territorial y ordenación de plantas que permiten aquellos índices serán especialmente útiles para efectuar interpretaciones y aplicaciones fitogeográficas bastante afinadas.

PAlabras Clave: Geografía de las plantas; escala local; ocupación de la flora; riqueza vascular; La Alcarria Occidental. 


\begin{abstract}
In order to clarify biogeographic concepts and know in detail vascular richness, a survey method at local scale is proposed. This employs the $1 \times 1 \mathrm{~km}$ UTM grid cell (ED50) as basic information unit and a $10 \times 10 \mathrm{~km}$ grid cell as the main spatial unit. Two UTM grid cells belonging to the same natural region (Western "La Alcarria") are analyzed in different sectors of it (30TWL02 and 30TVK86) to propitiate their comparison. The results were: a) discrimination of two plant groups due to phenological differences, b) design and formulation of two new indices that quantify the floral cover, c) detection of high values of vascular richness (around to a thousand of taxa in $100 \mathrm{~km}^{2}$ ); and d) exhibition of a Checklist of plants whose distribution can be mapped with resolution of $1 \mathrm{~km}^{2}$. The spatial comparison and plant ordering that allow those indices will be very useful for making finer interpretations and phytogeographical applications.
\end{abstract}

KEY WORDS: Plant Geography; local scale; floral cover; vascular richness; Western "La Alcarria".

\title{
RÉSUMÉ
}

Afin de clarifier concepts biogéographiques et connaître la richesse floristique détaillée, on présente une méthode de rélévé a l'échelle local qui emploie le quadrillage U.T.M. de $1 \times 1 \mathrm{~km}$ (ED50) comme unité basique d'information et de 10x10 km comme territoire d'étude. Deux carrés de $10 \mathrm{~km}$ de côté qui appartienent à la même région naturelle ("La Alcarria" de l'Ouest) sont choisis, mais situés dans différents secteurs (30TWL02 et 30TVK86) pour faciliter la comparaison. Les résultats ont été les suivants: a) distinction de deux groupements floristiques par differences phénologiques, b) conception et formulation de deux nouveaux indices qui permettent de quantifier l'occupation de la flore, c) détection des chiffres élevés de richesse vasculaire (environ mille taxons dans $100 \mathrm{~km}^{2}$ ); et d) exposition d'un Catalogue des plantes dont la distribution est représentable avec une résolution de $1 \mathrm{~km}^{2}$. La comparaison territoriale et le rangement des plantes que ceux indices permettent seront très utiles pour faire des interprétations et applications phytogéographiques bien justes.

MotS CLÉs: Géographie des plantes; échelle local; occupation de la flore; richesse vasculaire; "La Alcarria" de l'Ouest. 